\title{
Q-Adaptation of UKF Algorithm for Estimation of the Autonomous Underwater Vehicles Dynamics
}

\author{
Chingiz Hajiyev ${ }^{1}$, Halil Ersin Soken ${ }^{2}$ \\ ${ }^{1}$ Istanbul Technical University, Aeronautics and Astronautics Faculty \\ Maslak 34469, Istanbul, Turkey \\ cingiz@itu.edu.tr \\ ${ }^{2}$ Japan Aerospace Exploration Agency (JAXA), Institute of Space and Astronautical Science (ISAS) \\ Kanagawa, Japan \\ ersin_soken@ac.jaxa.jp
}

\begin{abstract}
Accurate estimation for the pose and orientation of the autonomous underwater vehicles (AUVs) is necessary in most of the applications. This is same for both the missile type AUVs or the vehicles used for research purposes deep in the sea or ocean. A nonlinear version of the Kalman Filter as the unscented Kalman filter (UKF) gives satisfactory estimation results for this purpose in the normal operation conditions. However, in the deep sea, changes in the environment (process noise) either instantaneously or periodically are very likely. In such case, the UKF must be adapted to become robust against such changes and provide accurate estimation results even in this case. In this study, we propose process noise covariance matrix adaptation (Q-adaptation) for the UKF algorithm. The main aim is to make the algorithm adaptive against the changes in the process noise covariance. The Adaptive UKF (AUKF) estimates the AUVs dynamics. We investigate the performance of the algorithm when the process noise increases and decreases, which are very likely as a result of changes in the AUV dynamics in different environments. The results are compared with those of a non-Q-adaptive version of the UKF algorithm.
\end{abstract}

Keywords: Autonomous Underwater Vehicle, Estimation, Q-Adaptation, Adaptive Kalman Filtering, Scale Factor.

\section{Introduction}

Researches on underwater systems have gained an immense interest during the last decade with applications in many different fields. Significant number of autonomous underwater vehicles (AUVs) has been developed for performing a wide range of scientific missions for deep sea research. The AUVs require a precise navigation system for localization, positioning, path tracking, guidance, and control during long period of duty cycles. To develop an accurate and robust navigation and control system for an AUV, fault tolerant estimation of the AUV dynamics is a crucial requirement.

Since it was proposed, Kalman filter has been widely used as a method to estimate the AUV dynamics parameters [1] and different Kalman filter types have been tested for that purpose. As a known fact, dynamics parameters of an autonomous underwater vehicle (AUV) cannot be estimated by linear Kalman filters because of the inherent nonlinear dynamics and kinematics. In such case, the Extended Kalman Filter (EKF) may be used instead. The EKF can estimate motion dynamics parameters of an AUV, which has typical navigation sensor outfits such as compass, pressure depth sensor, and some class of inertial navigation system (INS) [2]. However, mandatory linearization phase of the EKF procedure may cause the filter to diverge and usually limits the filter's achievable estimation accuracy $[3,4]$.

Unscented Kalman Filter (UKF) is a newer Kalman filtering technique compared to the EKF and in case of nonlinear dynamics it affords considerably more accurate estimation results. The essence of the UKF is a deterministic sampling technique that we use for calculating a minimum number of sample points [4]. These sample points enable an estimation accuracy of third-order for Gaussian data compared to the first-order accuracy of the EKF. As a result the UKF has become one of the preferred filtering algorithms for the AUV dynamics estimation as well [5].

A drawback of the traditional Kalman filtering algorithms including the UKF, they are not robust against the environment changes. In case of such changes the estimation accuracy of the filter deteriorates and if the faults are long lasting, the filter may diverge. Specifically for the AUVs, harsh operation conditions and anomalies in the deep water make 
such circumstances very likely. This situation leads us to a search for an Adaptive UKF (AUKF) that we can use for dynamics estimation of an AUV.

Various algorithms for adapting the Kalman filter have been proposed starting with the earlier publications by Mehra $[6,7]$. One of these ideas is to scale the covariances of the filter. The parameters that are used for scaling can be calculated using different means [8-10]. This idea is also applicable to the UKF adaptation. In [11] we proposed an integrated algorithm which adapts both the measurement and process noise covariance matrices of the UKF for satellite attitude estimation.

In this paper, we adapt the UKF algorithm that we use for estimating the dynamics of an AUV by using a straightforward approach. Different than our previous researches on adaptive Kalman filtering, which examined a linear problem with both linear process and measurement models [9], and a nonlinear problem with both nonlinear process and measurement models [11], this time the system equation is nonlinear but the measurement model is linear. Hence we use a special form of the UKF. Besides we apply the method for the AUV dynamics estimation for the first time and show that the process noise covariance of the filter can be easily adapted to ensure both the stability and accuracy in this special from with linear measurement model. Since the measurement model of the UKF is linear the filter adaptation is similar to the one for a linear KF and simpler compared to the exiting AUKF methods for underwater vehicles.

Different than the existing works such as $[12,13]$, which require parallel two filters running to both estimate the AUV dynamics and adapt the filter, the proposed algorithm has only one filter running. Thus it does not require a cumbersome additional computational load opposed to the two-filter based adaptive algorithms $[12,13]$. There are other single-filter based adaptive algorithms proposed for AUV state estimation in the literature [14]. However they have other drawbacks such as suffering from instability issue, which appears whenever the adapted noise covariance matrix lose positive semidefiniteness. In contrast in the proposed algorithm the stability is secured by 1) adapting the filter only when necessary and using the optimal process noise covariance throughout the rest of the estimation process; 2) using the covariance scaling approach in which the adaptive factor takes always positive values.

\section{Autonomous Underwater Vehicle Mathematical Model}

AUV modeling is fairly complicated, and an exact analysis is only possible by including the underlying infinite dimensional dynamics of the surrounding fluid (sea water). While this can be done by using partial differential equations in Computational Fluid Dynamics (CFD) computer tools, it still involves a formidable computational burden, and is infeasible for most practical applications.

AUVs motion with 6 degrees of freedom (6DOF) is necessary to determine the position and orientation of a rigid body $[15,16]$. The first three coordinates and their time derivatives are of translational motion along the $\mathrm{x}, \mathrm{y}$ and $\mathrm{z}$-axes, while the last three coordinates $(\phi, \theta, \psi)$ and their time derivatives are used to describe the orientation and rotational motion (See Fig.1).

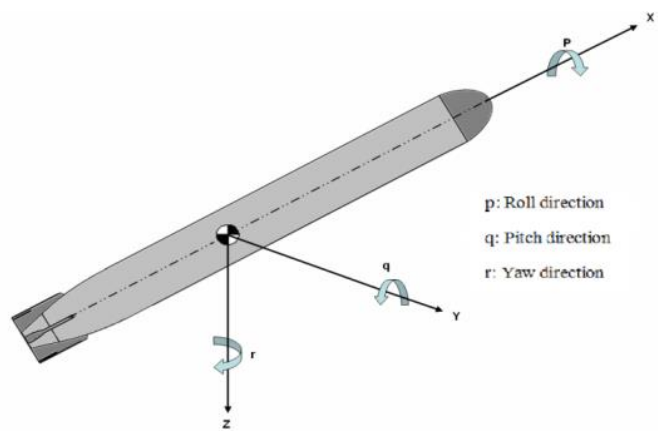

Fig. 1: 6DOF AUV Angular and Translational Motions.

In this study, 6DOF AUV equations of motion are derived as below [15]:

Surge or translational motion along the $\mathrm{x}$-axis:

$$
\begin{aligned}
& X=X_{\dot{u}} \dot{u}+m\left[-\dot{u}-z_{G} \dot{q}+y_{G} \dot{r}\right]+X_{u u} u|u|+\left(X_{w q}-m\right) w q+\left(X_{q q}+m x_{g}\right) q^{2} \\
& +\left(X_{v r}+m\right) v r+\left(X_{r r}+m x_{g}\right) r^{2}-m z_{g} p r-(W-B) \sin \theta+X_{p r o p}
\end{aligned} .
$$


Sway or translational motion along the y-axis:

$$
\begin{aligned}
& Y=Y_{\dot{v}} \dot{v}+Y_{\dot{r}} \dot{r}+m\left[-\dot{v}+z_{g} \dot{p}-x_{g} \dot{r}\right]+Y_{u v} u v+\left(Y_{w p}+m\right) w p+\left(Y_{u r}-m\right) u r- \\
& \left(m z_{g}\right) q r+\left(Y_{p q}-m x_{g}\right) p q+Y_{v v} v|v|+Y_{r r} r|r|+(W-B) \cos \theta \sin \phi+Y_{u u \delta_{r}} u^{2} \delta_{r}
\end{aligned}
$$

Heave or translational motion along the z-axis:

$$
\begin{aligned}
& Z=Z_{\dot{w}} \dot{w}+Z_{\dot{q}} \dot{q}+m\left[-\dot{w}+x_{g} \dot{q}-y_{g} \dot{p}\right]+\left(Z_{u q}+m\right) u q+\left(Z_{v p}-m\right) v p+\left(m z_{g}\right) p^{2}+ \\
& Z_{u w} u w+\left(m z_{g}\right) q^{2}+\left(Z_{r p}-m x_{g}\right) r p+Z_{w w} w|w|+Z_{q q} q|q|+(W-B) \cos \theta \cos \phi+Z_{u u \delta_{e}} u^{2} \delta_{e}
\end{aligned} .
$$

Roll or rotation about the $\mathrm{x}$-axis:

$$
\begin{aligned}
& K=\mathrm{mz}_{g} \dot{\mathrm{u}}-m y_{g} \dot{r}-\left(I_{x x}-K_{\dot{p}}\right) \dot{p}+\left(m y_{g}\right) \dot{w}-\left(I_{z z}-I_{y y}\right) q r-\left(m z_{g}\right) w p+\left(m z_{g}\right) u r \\
& -\left(z_{g} W-z_{b} B\right) \cos \theta \sin \psi-\left(y_{g} W-y_{b} B\right) \cos \theta \cos \psi+K_{p p} p|p|+K_{p r o p}
\end{aligned} .
$$

Pitch or rotation about the y-axis:

$$
\begin{aligned}
& M=-m z_{g} \dot{u}+\left(m x_{g}+M_{\dot{w}}\right) \dot{w}-\left(I_{y y}-M_{\dot{q}}\right) \dot{q}+\left(M_{r p}+I_{z z}-I_{x x}\right) r p+\left(m z_{g}\right) v r \\
& -\left(m z_{g}\right) w q+\left(M u q-m x_{g}\right) u q+M_{u w} u w+\left(M_{v p}+m x_{g}\right) v p+M_{q q} q|q|+ \\
& M_{w w} w|w|-\left(z_{g} W-z_{b} B\right) \sin \theta-\left(x_{g} W-x_{b} B\right) \cos \theta \cos \psi+M_{u u \delta_{e}} u^{2} \delta_{e}
\end{aligned} .
$$

Yaw or rotation about the z-axis:

$$
\begin{aligned}
& N=m y_{g} \dot{u}+\left(N_{\dot{v}}-m x_{g}\right) \dot{v}+\left(N_{\dot{r}}-I_{z z}\right) \dot{r}+\left(N p q+I_{x x}-I_{y y}\right) p q+\left(N_{w p}-m x_{g}\right) w p \\
& +\left(N_{u r}-m x_{g}\right) u r+N_{u v} u v+N_{r r} r|r|+N_{v v} v|v|+\left(x_{g} W-x_{b} B\right) \cos \theta \sin \psi+\left(y_{g} W-y_{b} B\right) \sin \theta+N_{u u \delta_{r}} u^{2} \delta_{r}
\end{aligned} .
$$

where; X, Y, Z, K, M, and N represent the resultant forces and moments with respect to the body-fixed coordinates. Details about the physically based model parameters of AUV above and their definitions are given in [15].

\section{Unscented Kalman Filter for AUV Dynamics Estimation}

The UKF uses sigma points that are calculated based on a priori mean and covariance of the state to represent the state distribution. It is founded on the intuition that it is easier to approximate a probability distribution than it is to approximate a nonlinear function or transformation [3, 4]. In this sense, it offers a derivative-free alternative to the EKF with a more accurate approximation to nonlinear dynamics than the linearization of the Taylor series.

The initial step for the UKF is the determination of $2 n+1$ sigma points with a mean of $\hat{x}(k \mid k)$ and a covariance of $P(k \mid k)$. For an $n$ dimensional state vector, these sigma points are obtained by,

$$
\begin{gathered}
x_{0}(k \mid k)=\hat{x}(k \mid k), \\
x_{i}(k \mid k)=\hat{x}(k \mid k)+(\sqrt{(n+\kappa) P(k \mid k)})_{i},
\end{gathered}
$$




$$
x_{i+n}(k \mid k)=\hat{x}(k \mid k)-(\sqrt{(n+\kappa) P(k \mid k)})
$$

where, $x_{0}(k \mid k), x_{i}(k \mid k)$ and $x_{i+n}(k \mid k)$ are sigma points, $n$ is the state number and $\kappa$ is the scaling parameter which is used for fine tuning and the heuristic is to chose that parameter as $n+\kappa=3$. Also, $i$ is given as $i=1 \mathrm{~K} n$ and $(\cdot)_{i}$ corresponds to the $i^{\text {th }}$ column of the matrix.

Next each sigma point is transferred by using system dynamics,

$$
x_{i}(k+1 \mid k)=f\left[x_{i}(k \mid k), k\right] . \quad i=1 \mathrm{~K} n .
$$

These transformed values are used to get the predicted mean and the covariance,

$$
\begin{gathered}
\hat{x}(k+1 \mid k)=\frac{1}{n+\kappa}\left\{\kappa x_{0}(k+1 \mid k)+\frac{1}{2} \sum_{i=1}^{2 n} x_{i}(k+1 \mid k)\right\}, \\
P(k+1 \mid k)=\frac{1}{n+\kappa}\left\{\kappa\left[x_{0}(k+1 \mid k)-\hat{x}(k+1 \mid k)\right]\left[x_{0}(k+1 \mid k)-\hat{x}(k+1 \mid k)\right]^{T}\right. \\
\left.+\frac{1}{2} \sum_{i=1}^{2 n}\left[x_{i}(k+1 \mid k)-\hat{x}(k+1 \mid k)\right]\left[x_{i}(k+1 \mid k)-\hat{x}(k+1 \mid k)\right]^{T}\right\}+Q(k) .
\end{gathered}
$$

Here, $\hat{x}(k+1 \mid k)$ is the predicted mean, $Q(k)$ is the process noise covariance and $P(k+1 \mid k)$ is the predicted covariance.

Further steps of the UKF for the AUV dynamics estimation problem are similar to those of a linear Kalman filter since the measurement model is linear. In these steps we do not need to use the sigma points anymore. This simplifies the procedure and also reduces the computational load. First, the predicted observation vector is,

$$
\hat{y}(k+1 / k)=H(k+1) \hat{x}(k+1 / k)
$$

where $H(k+1)$ is the measurement matrix.

After that, observation covariance matrix and the cross correlation matrix are determined as,

$$
\begin{gathered}
P_{y y}(k+1 / k)=H(k+1) P(k+1 / k) H^{T}(k+1), \\
P_{x y}(k+1 / k)=P(k+1 / k) H^{T}(k+1)
\end{gathered}
$$

Following part is the update phase of UKF algorithm. At that phase, first by using measurements, $y(k+1)$, residual term (or innovation sequence) $v(k+1)$ is found as the difference between the actual observation and the predicted observation:

$$
v(k+1)=y(k+1)-\hat{y}(k+1 \mid k),
$$

The innovation covariance is,

$$
P_{v v}(k+1 \mid k)=P_{y y}(k+1 \mid k)+R(k+1)=H(k+1) P(k+1 / k) H^{T}(k+1)+R(k+1)
$$


Here $R(k+1)$ is the measurement noise covariance matrix.

The optimal Kalman gain is computed as

$$
K(k+1)=P_{x y}(k+1 \mid k) P_{v v}^{-1}(k+1 \mid k) .
$$

At last, the updated state and covariance matrix are determined by,

$$
\begin{aligned}
& \hat{x}(k+1 \mid k+1)=\hat{x}(k+1 \mid k)+K(k+1) v(k+1), \\
& P(k+1 \mid k+1)=P(k+1 \mid k)-P_{x y}(k+1 / k) P_{v v}^{-1}(k+1 \mid k) P_{x y}{ }^{T}(k+1 / k),
\end{aligned}
$$

or

$$
P(k+1 \mid k+1)=P(k+1 \mid k)-K(k+1) P_{v v}(k+1 \mid k) K^{T}(k+1) .
$$

Here, $\hat{x}(k+1 \mid k+1)$ is the estimated state vector and $P(k+1 \mid k+1)$ is the estimated covariance matrix.

\section{Process Noise Covariance Adaptation for the UKF Algorithm}

A Q-adaptive algorithm must be introduced such that the filter is insensitive to process noise changes and corrects estimation process without affecting the good estimation behavior. Adaptive algorithm affects the characteristics of the filter only when the condition of the process noise does not correspond to the model used in the synthesis of the filter. Otherwise the filter works with regular algorithm (7)-(21) in an optimally.

Adaptation occurs as a change in the predicted covariance. First, let us rewrite (12) as;

$$
P(k+1 \mid k)=P^{*}(k+1 \mid k)+Q(k) .
$$

So $P^{*}(k+1 \mid k)$ is the predicted covariance without the additive process noise. In order to adapt the covariance an adaptive scaling factor is put into the covariance matrix of the innovation sequence;

$$
P_{v v}(k+1 \mid k)=H(k+1) P^{*}(k+1 / k) H^{T}(k+1)+\Lambda(k)\left[H(k+1) Q(k) H^{T}(k+1)\right]+R(k+1) .
$$

Here $\Lambda(k)$ is the adaptive process noise scale factor.

The left hand side of (23) represents the real filtration error while the right hand side is the accuracy of the innovation sequence known as a result of priori information [11]. When the predicted observation vector $\hat{y}(k+1 / k)$ is reasonably different from the measurement vector, $y(k+1)$, the real filtration error exceeds the theoretical one. Hence, the gain matrix must be fixed hereafter by the use of adaptive algorithm and the scale factor $\Lambda(k)$ [11]. To calculate the system noise scale factor, the equality of

$$
\operatorname{tr}\left[v(k+1) v^{T}(k+1)\right]=\operatorname{tr}\left[H(k+1) P^{*}(k+1 / k) H^{T}(k+1)\right]+\Lambda(k) \operatorname{tr}\left[H(k+1) Q(k) H^{T}(k+1)\right]+\operatorname{tr}[R(k+1)]
$$

is used. Here $\operatorname{tr}($.$) is the trace of the related matrix. Taking into consideration that$

$$
\operatorname{tr}\left[v(k+1) v^{T}(k+1)\right]=v^{T}(k+1) v(k+1),
$$


the single scale factor $\Lambda(k)$ is determined from the equality (24) as

$$
\Lambda(k)=\frac{v^{T}(k+1) v(k+1)-\operatorname{tr}\left[H(k+1) P^{*}(k+1 / k) H^{T}(k+1)\right]-\operatorname{tr}[R(k+1)]}{\operatorname{tr}\left[H(k+1) Q(k) H^{T}(k+1)\right]}
$$

We can conclude that if the process noise covariance change, $\Lambda(k)$ will change and so affect the Kalman gain matrix as

$$
K(k+1)=P_{x y}(k+1 \mid k) \times\left\{H(k+1) P^{*}(k+1 / k) H^{T}(k+1)+\Lambda(k)\left[H(k+1) Q(k) H^{T}(k+1)\right]+R(k+1)\right\}^{-1} .
$$

According to the proposed approach the gain matrix of UKF is changed when the following condition is valid

$$
\begin{aligned}
& \operatorname{tr}\left[v(k+1) v^{T}(k+1)\right] \geq \operatorname{tr}\left[H(k+1) P^{*}(k+1 / k) H^{T}(k+1)\right] \\
& +\operatorname{tr}\left[H(k+1) Q(k) H^{T}(k+1)\right]+\operatorname{tr}[R(k+1)]
\end{aligned}
$$

If the left side of the expression (28) is greater than the right side, the scale factor value $\Lambda(k)$ will increase. This corresponds to the beginning of adaptation of filter. Consequently, the innovation covariance (equation (23)) increases, and the filter gain matrix $K(k+1)$ (equation (27)) decreases, which will cause strengthening of the corrective influence of innovation sequence in the estimation algorithm and approach the estimation value $\hat{x}(k+1 \mid k+1)$ to the actual value $x(k+1)$. This will lead to the decrease of innovation sequence $v(k+1)$ (equation (16)) and adaptive factor $\Lambda(k)$, weakening of the corrective influence of innovation sequence, etc. Consequently, an adaptive scheme is performed to adjust the process noise covariance matrix in response to the changing environment. As a result, the changes in the process noise covariance are corrected by the system, without affecting the good estimation behaviour.

Remark that, due to the scale factor, the covariance of the estimation error of the AUKF increases in comparison with the regular UKF. Therefore, the adaptive algorithms are used only in case of change in the process noise covariance and in all other cases the optimal UKF gain is used.

\section{Simulation Results of the Q-Adaptive UKF}

During simulations for testing the Q-adaptive UKF algorithm, the process noise covariance matrix is changed temporarily for a period in between $40^{\text {th }}$ and $45^{\text {th }} \mathrm{s}$. At first this change is simply formed by multiplying the covariance with a constant and increasing the covariance for this time interval. As is apparent from Figs. 2 and 3, the proposed Q-adaptive UKF is not affected significantly by the change in the process noise covariance, and ensures accurate estimates for the whole process by decreasing the adaptive factor. 

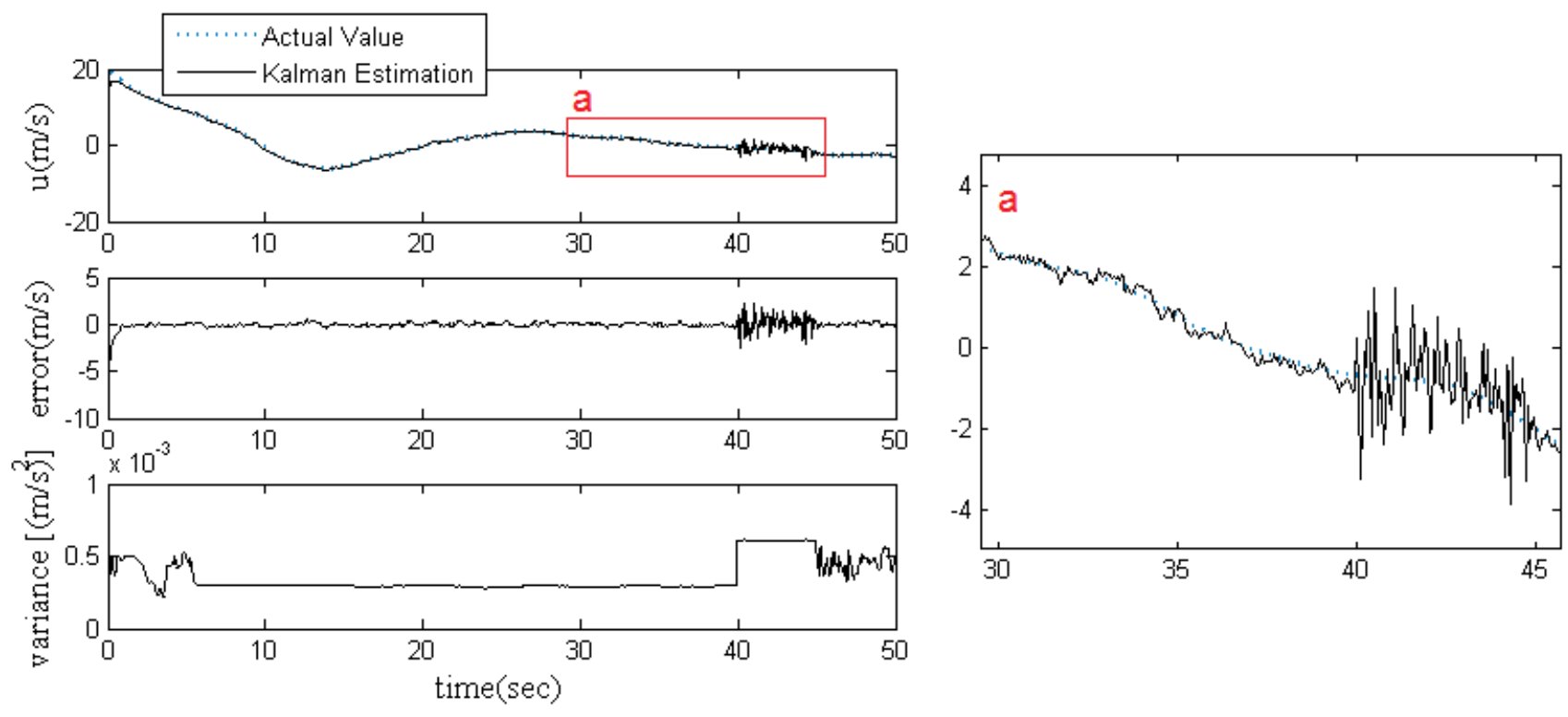

Fig. 2: Estimation of u velocity term via Q-adaptive UKF in the case of temporary change in the process noise covariance. Subfigure (a) zooms to the indicated part on the main figure.

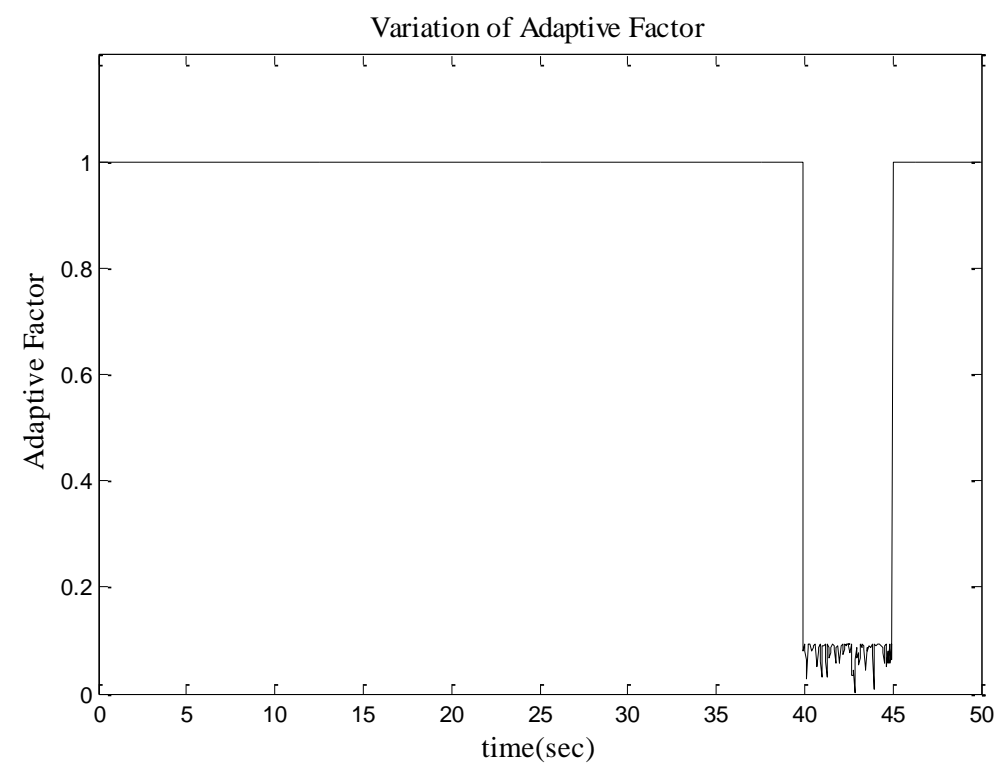

Fig.3: Behavior of adaptive factor when Q matrix increases.

In Fig. 3, variation of the adaptive factor for Q scaling is presented for the period within the $40^{\text {th }}$ and $45^{\text {th }}$ seconds. As expected, it takes smaller values than 1 so as to reduce the process noise covariance and then changes in a relevant way with the changing characteristics of the estimation process. Another fact is the Q-adaptation scheme works in a similar manner when there is a decrease in the process noise covariance, which may risk the stability of the algorithm, and increases the covariance to match with the real filtration error. In a case we decrease the Q matrix significantly $\left(10^{-10}\right.$ times smaller Q values than the optimal one) the adaptive algorithm reacts by increasing the adaptive factor and ensures both the estimation accuracy and filter stability (Figs.4-5). 

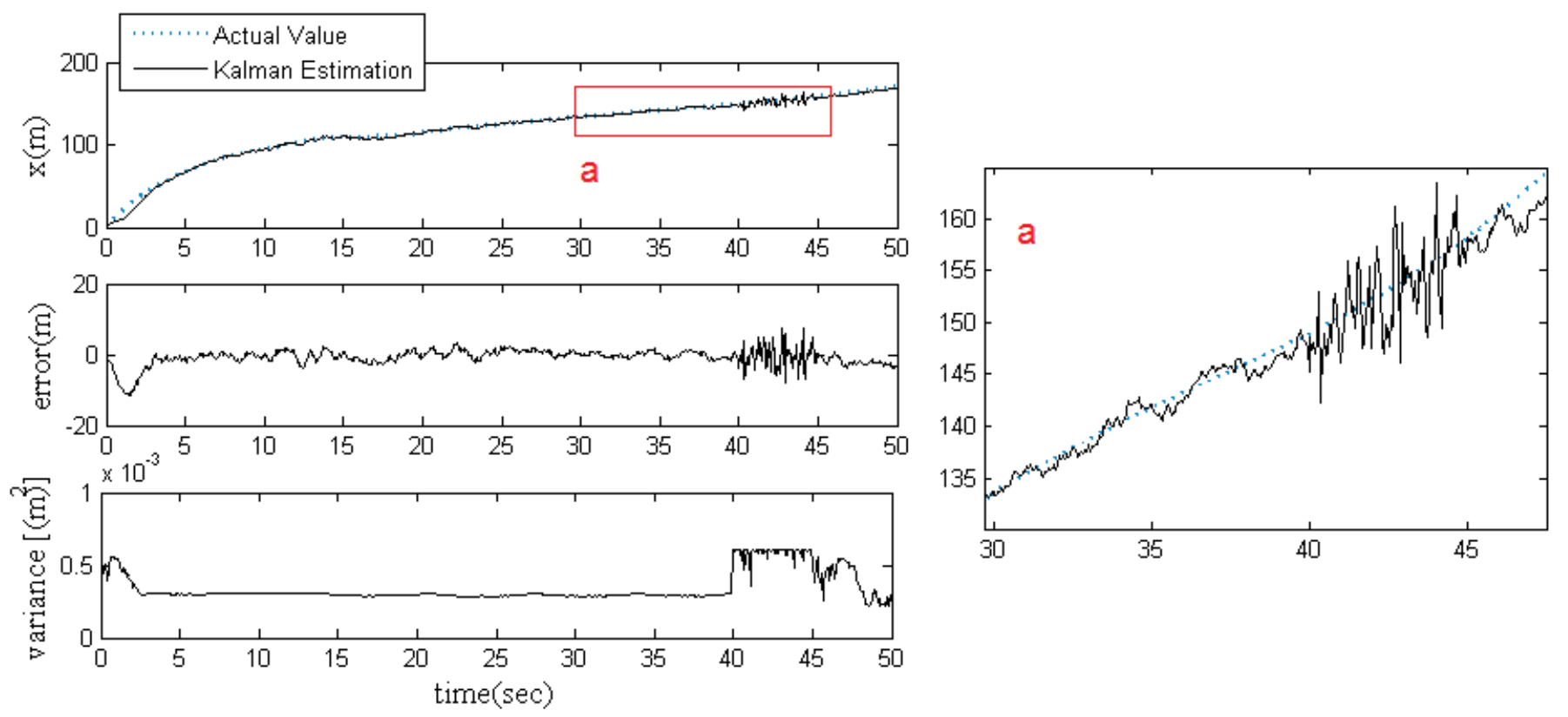

Fig. 4: Estimation of X-position via Q-adaptive UKF in the case of temporary change of the process noise covariance. Subfigure (a) zooms to the indicated part on the main figure.

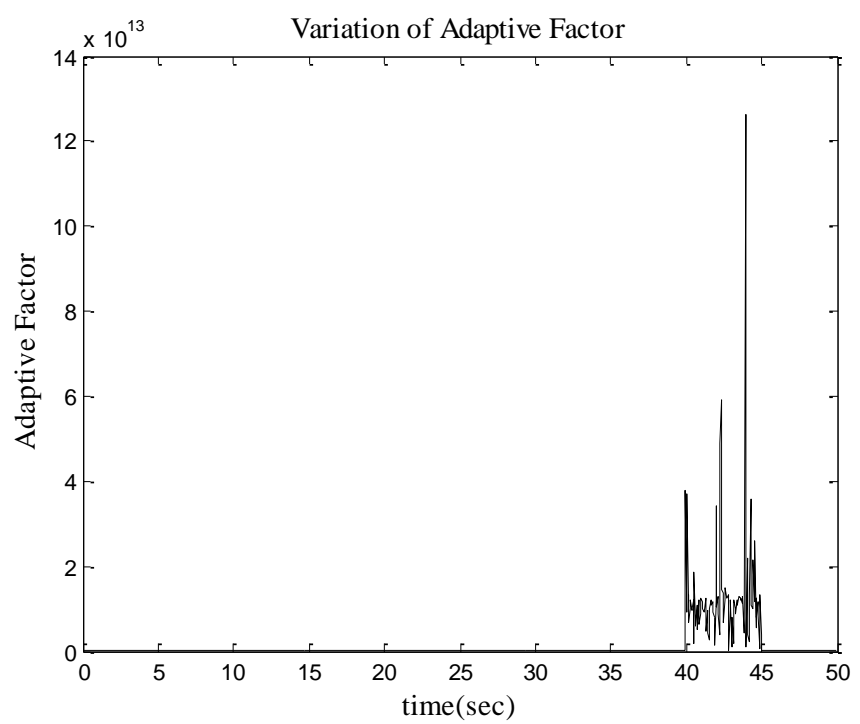

Fig. 5: Behavior of adaptive factor in case Q matrix decreases.

\section{Conclusions}

There are many difficulties for estimating the dynamics of an autonomous underwater vehicle in operation. One of these difficulties is the changes in the environment (process noise) that may deteriorate the estimator's accuracy. In such case the estimator, which is implemented onboard the AUV, must be adapted to make it robust against environment changes and provide accurate estimation results even in this case. In this study, we adapted the UKF algorithm that we use for estimating the dynamics of an AUV and propose process noise covariance matrix adaptation (Q-adaptation) for the UKF algorithm. The main aim is to make the algorithm adaptive against the changes in the process noise covariance. The Adaptive UKF estimates the AUVs dynamics. The numerical results confirm that the adaptive UKF gives better results than the regular UKF in the case of environment changes. 
The results of this study show that it is not possible to get precise estimation results by the regular UKF if the model for the process noise covariance disagrees with the real value as a result of some change. An adaptive scheme to adjust the process noise covariance matrix in response to the changing environment is proposed. The presented adaptive UKF algorithm is not affected by those changes and secures its good estimation characteristic all the time. The additional computational load of the adaptation is negligible.

\section{References}

[1] A. Lammas, K. Sammut and F. He, "6-DoF Navigation Systems for Autonomous Underwater Vehicles," Mobile Robots Navigation, Alejandra Barrera, Ed. InTech Publishing, 2010, pp.457-483.

[2] L. Stutters, H. Liu, C. Tiltman and D. J. Brown, "Navigation Technologies for Autonomous Underwater Vehicles," IEEE Trans. Syst., Man, Cyber.: Appl. Rev. , vol. 38, No. 4, pp.581-589, 2008.

[3] S. J. Julier, J. K. Uhlmann and H. F. Durrant-Whyte, "A new approach for filtering nonlinear systems," Proc. of American Control Conference, Seattle, USA, 1995, vol. 3, pp.1628-1632.

[4] S. J. Julier, J. K. Uhlmann and H. F. Durrant-Whyte, "A new method for the nonlinear transformation of means and covariances in filters and estimators," IEEE Trans. Autom. Control, vol. 45, pp. 477-482, 2000.

[5] J. Kim, K. Kim, H. S.Choi, W. Seong and K-Y Lee, "Estimation of Hydrodynamic Coefficients for an AUV Using Nonlinear Observers," IEEE J. Ocean. Eng., vol. 27, No. 4, pp. 830-840, 2002.

[6] R. K., Mehra, "On the Identification of Variances and Adaptive Filtering," IEEE Trans. Automatic Cont., vol. 15, No.2, pp.175-184, 1970.

[7] R. K., Mehra, “Approaches to Adaptive Filtering," IEEE Trans. on Automatic Cont., vol. 17, No.10, pp. 693-698, 1972.

[8] C. Hajiyev, "Adaptive filtration algorithm with the filter gain correction applied to integrated INS/Radar altimeter," Proc. IMechE, Part G: J. Aerosp. Eng., vol. 221, pp. 847-885, 2007.

[9] C. Hajiyev and H. E. Soken, "Robust Estimation of UAV Dynamics in the Presence of Measurement Faults," $J$. Aerosp. Eng., vol. 25, No. 1, pp. 80-89, 2012.

[10] C. H. Kang, S. Y. Kim and C. G. Park, "A GNSS interference identification and tracking based on adaptive fading Kalman filter, Proc. 19th IFAC World Congress, 2014, vol.19, pp. 3250-3255.

[11] C. Hajiyev and H. E. Soken, "Robust adaptive unscented Kalman filter for attitude estimation of pico satellites," International Journal of Adaptive Control and Signal Processing, vol. 28, No. 2, pp. 107-120, 2014.

[12] K. Liu, J. Li, W. Guo, P. Zhu and X. Wang "Navigation of a class of underwater vehicle based on adaptive unscented Kalman filter algorithm," J. Cent. South Univ, vol. 21, pp.550-557, 2014.

[13] W. Li, Y. Li, S. Ren and X. Feng "Tracking and underwater manoeuvring target using an adaptive Kalman filter," Proc. IEEE Tencon, pp.1-4, 2013.

[14] Y. Lin, K. Liu and X. Wang, "An adaptive square-root unscented Kalman filter for underwater vehicle navigation," Proc. of IEEE Int. Conf. on Mechatronics and Automation, pp.717-722. 2014.

[15] T. Prestero, "Verification of a Six-Degree of Freedom Simulation Model for the REMUS Autonomous Underwater Vehicle," M.Sc. Thesis in Ocean and Mechanical Engineering, MIT, USA, 2001.

[16] M. Dinc and C. Hajiyev, "Integration of navigation systems for autonomous underwater vehicles," J.Marine Engineering \& Technology, vol. 14, no.1, pp.32-43, 2015. 